\title{
Generating stable attosecond x-ray pulse trains with a mode-locked seeded free-electron laser
}

\author{
Chao Feng, ${ }^{1,2}$ Jianhui Chen, ${ }^{1}$ and Zhentang Zhao ${ }^{1, *}$ \\ ${ }^{1}$ Shanghai Institute of Applied Physics, Chinese Academy of Sciences, Shanghai 201800, China \\ ${ }^{2}$ Graduate University of Chinese Academy of Sciences, Beijing 100049, China
}

(Received 15 March 2012; published 14 August 2012)

\begin{abstract}
Generation of attosecond x-ray pulses is attracting much attention within the x-ray free-electron laser (FEL) user community. We propose a novel scheme for the generation of coherent stable attosecond x-ray pulse trains in a seeded FEL, via a process of mode-locked amplification. Three modulators and two chicanes are used for generating separated attosecond scale microstructures in the electron beam using the beam echo effect. Such electron beam will produce high harmonic radiation with a comb of longitudinal modes at the very beginning of the radiator. By using a series of spatiotemporal shifts between the copropagating radiation and electron beam in the radiator, all these modes can be preserved and amplified to saturation. Using a representative realistic set of parameters, three-dimensional simulation results show that trains of 200 attosecond soft x-ray pulses with stable peak powers at gigawatt level can be generated directly from ultraviolet seed lasers. The even spacing between the attosecond pulses can be easily altered from subfemtosecond to tens of femtoseconds by slightly changing the wavelength of one seed laser.
\end{abstract}

DOI: 10.1103/PhysRevSTAB.15.080703

PACS numbers: 41.60.Cr

\section{INTRODUCTION}

Ultrashort x-ray sources have the potential to open new regimes in atomic and electronic processes, benefiting widespread fields in physics, chemistry, and biology. There have been many proposals for generation of attosecond pulses using the high-gain free-electron laser (FEL) [1-11]. Some of these schemes [2-8] that rely on the self-amplified spontaneous emission (SASE) mechanism to generate attosecond $\mathrm{x}$-ray pulses may suffer from statistic fluctuations because SASE originates from electron beam shot noise. An alternative way to overcome this problem is using the seeded FEL schemes such as highgain harmonic generation [12] or echo-enabled harmonic generation (EEHG) $[13,14]$ to generate stable attosecond pulses [9-11]. As the output pulse lengths produced by these seeded FEL schemes are usually determined by the slippage length in the radiator, quite short radiators ( $\sim 10$ periods) are often adopted, which means that no exponential growth is expected in the radiator and results in a relatively low output power.

Attosecond pulse trains (APTs) have proven a valuable tool in atomic and molecular science [15-20]. It has been proposed that the APTs can also be generated by the SASE FEL using a mode-locked amplifier [8], which consists of a system of undulator-chicane modules. In this amplifier, a comb of longitudinal modes can be synthesized by the

\footnotetext{
*Corresponding author. zhaozt@sinap.ac.cn

Published by the American Physical Society under the terms of the Creative Commons Attribution 3.0 License. Further distribution of this work must maintain attribution to the author(s) and the published article's title, journal citation, and DOI.
}

optics-free technique applying a series of temporal shifts between the copropagating radiation and electron bunch. The neighboring modes may be locked in phase by introducing an interaction modulation at the mode spacing, for example, an energy modulation induced by a seed laser interacting with the electron beam in a modulator. As with the conventional atomic laser, this mode locking modifies the temporal envelop of the output field from continuous wave to a train of short pulses, periodically spaced by the wavelength of the seed laser. It has been shown in Ref. [21] that the spectral brightness of the radiation can be increased by replacing the beam energy modulation with a beam current modulation of the same period. However, as the radiation originates from the shot noise of the electron beam, these schemes may still have large shot-to-shot output power fluctuations and usually need a large number of undulator-chicane modules to produce a clean series of short pulses [22]. Moreover, the spacing between the attosecond pulses is limited by the wavelength of the seed laser.

EEHG is a very promising candidate for the generation of coherent short wavelength FEL with a single stage of seeded FEL setup. The modulation mechanism of EEHG has been experimentally demonstrated both at the Next Linear Collider Test Accelerator (NLCTA) at SLAC National Accelerator Laboratory [23,24] and the Shanghai Deep-Ultra Violet FEL (SDUV-FEL) facility [25] recently. The echo signal has been successfully amplified by the long radiator of the SDUV-FEL [26]. The EEHG scheme seems an attractive way for the fully coherent soft x-ray light source. Several labs, including DESY [27], LBNL [28], and SINAP [29], proposed the development of soft x-ray FEL based on the EEHG principle.

In this paper, a novel scheme that combines the EEHG technique with the mode-locked technique is proposed to 


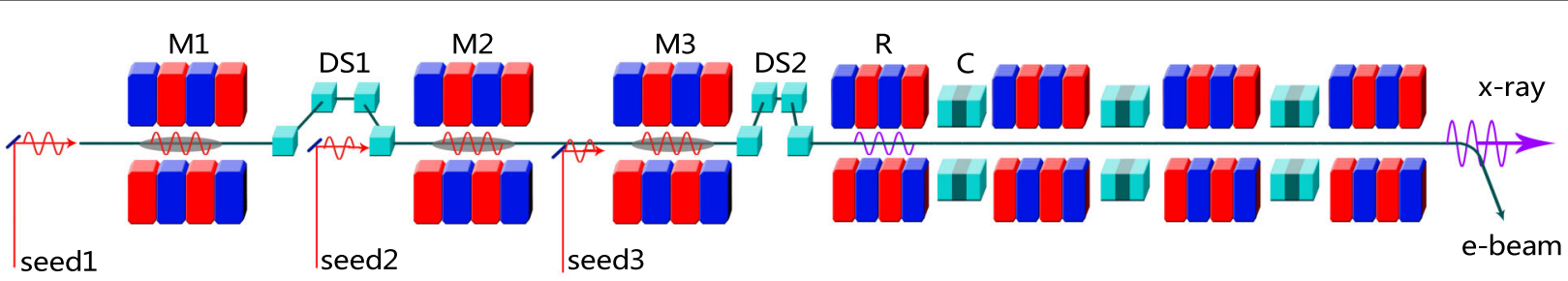

FIG. 1. Schematic of the proposed scheme for generation of attosecond x-ray pulse trains. (M: modulator; DS: dispersive section; $\mathrm{R}$ : radiator; $\mathrm{C}$ : chicane).

generate coherent, stable attosecond $\mathrm{x}$-ray pulse trains with GW level peak power directly from ultraviolet seed lasers. The principle of the mode-locked seeded FEL scheme is introduced in Sec. II. The analytical estimate is given, which shows the possibility of imprinting an attosecond microbunching structure into the electron beam. Section III gives an optimized design for a soft x-ray FEL based on the proposed scheme. Some practical constraints that may deteriorate the performance are studied in Sec. IV. Finally, we conclude in Sec. V.

\section{MODE LOCKING OF THE SEEDED FEL}

The proposed scheme consists of three modulators, two dispersion sections (DSs) and a mode-locked radiator, as shown in Fig. 1. Compared to the conventional EEHG scheme $[13,14]$, one more seed laser and modulator are added for tuning the spacing of the APT. The modulators and DSs are used for generation of density modulation at high harmonics with a relatively small initial energy modulation of the electron beam, which benefits from the beam echo effect [13]. The electron beam is first energy modulated by a seed laser (seed1) with wave number $k_{1}$ in the first modulator (M1) and then sent through a strong dispersion section (DS1), which makes the energy modulation induced in M1 macroscopically smeared out, but many well-structured beamlets appear in the phase space. This electron beam then interacts with two seed lasers (seed2 and seed3) with different wave numbers $k_{2}$ and $k_{3}$ to obtain sufficient energy modulations in M2 and M3, respectively. Meanwhile, the beating frequency $k=k_{2}-k_{3}$ occurs in the phase space of the electron beam. When $k$ is the subharmonic frequency of both $k_{2}$ and $k_{3}$ (i.e. $k=$ $k_{2} / m_{1}=k_{3} / m_{2}$, where $m_{1}$ and $m_{2}$ are integer numbers), the phase space will be periodic with a new energy modulation envelope period $\lambda_{s}=2 \pi / k$, which can be tuned to be much longer than the wavelengths of the seed lasers. After the electron beam passing through the second dispersive section (DS2), the energy modulation will be converted to density modulation and the echo signal occurs at some high harmonics of the seed lasers.

Following the notation of Ref. [14], we also assume an initial Gaussian beam energy distribution with an average energy $E_{0}$ and use the variable $p=\left(E-E_{0}\right) / \sigma_{E}$ for the dimensionless energy deviation of a particle, where $\sigma_{E}$ is the rms energy spread. After the passage through M1, the electron beam is modulated with the amplitude $A_{1}=$ $\Delta E_{1} / \sigma_{E}$, where $\Delta E_{1}$ is the energy modulation depth, and the dimensionless energy deviation of the electron beam becomes $p^{\prime}=p+A_{1} \sin \left(k_{1} z\right)$. Then the electron beam is sent through DS1 with the dispersive strength $R_{56(1)}$, and the distribution function can be written as

$$
f_{1}(\zeta, p)=\frac{N_{0}}{\sqrt{2 \pi}} \exp \left[-\frac{1}{2}\left[p-A_{1} \sin \left(\zeta-B_{1} p\right)\right]^{2}\right],
$$

where $\zeta=k_{1} z, z$ is the longitudinal coordinate in the beam, $N_{0}$ is the number of electrons per unit length of the beam, and $B_{1}=R_{56(1)} k_{1} \sigma_{E} / E_{0}$. Assume that the electron beam is then energy modulated in M2 and M3 with the same dimensionless amplitude $A_{2}=A_{3}$. After passing through DS2 with the dispersive strength $R_{56(2)}$, the longitudinal beam distribution evolves to

$$
\begin{aligned}
f_{2}(\zeta, p)= & \frac{N_{0}}{\sqrt{2 \pi}} \exp \left[-\frac{1}{2}\left\{p-A_{2} \sin \left(K_{1} \zeta-K_{1} B_{2} p+\phi\right)\right.\right. \\
& -A_{2} \sin \left(K_{2} \zeta-K_{2} B_{2} p+\phi^{\prime}\right) \\
& -A_{1} \sin \left[\zeta-\left(B_{1}+B_{2}\right) p\right. \\
& +A_{2} B_{1} \sin \left(K_{1} \zeta-K_{1} B_{2} p+\phi\right) \\
& \left.\left.\left.+A_{2} B_{1} \sin \left(K_{2} \zeta-K_{2} B_{2} p+\phi^{\prime}\right)\right]\right\}^{2}\right]
\end{aligned}
$$

where $B_{2}=R_{56(2)} k_{1} \sigma_{E} / E_{0}, \phi$ and $\phi^{\prime}$ are the phases of seed 2 and seed $3, K_{1}=k_{2} / k_{1}$ and $K_{2}=k_{3} / k_{1}$. Integration of this formula over $p$ gives the beam density $N$ as a function of $\xi, N(\zeta)=\int_{-\infty}^{\infty} f_{2}(\zeta, p) d p$.

The bunching factor at $n$th harmonic can be written as

$$
\begin{aligned}
b_{n}= & \mid \sum_{n_{1}=-\infty}^{\infty} \sum_{n_{2}=-\infty}^{\infty} J_{n 2}\left\{-n A_{2} B_{2}\right\} J_{n 3}\left[-n A_{2} B_{2}\right] \\
& \times J_{n 1}\left\{-A_{1}\left[n_{1} B_{1}+n B_{2}\right]\right\} e^{-1 / 2\left[n_{1} B_{1}+n B_{2}\right]^{2}} e^{i\left(n_{2} \phi+n_{3} \phi^{\prime}\right)} \mid,
\end{aligned}
$$

where $n_{1}, n_{2}$, and $n_{3}$ are integer numbers, and the harmonic number $n=n_{1}+K_{1} n_{2}+K_{2} n_{3}$. Analysis shows that the $n$th bunching factor attains its maximum when $n_{1}= \pm 1$ and $n_{2}=n_{3} . n_{1}$ is chosen to be -1 to make $B_{1}$ and $B_{2}$ have the same sign. For given $A_{1}$ and $A_{2}$, the maximal bunching factor is achieved when $B_{2}=\left(n_{2}+0.81 n_{2}^{1 / 3}\right) / n A_{2}$ and $B_{1}$ is the solution of 


$$
\begin{aligned}
& A_{1}\left\{J_{0}\left[A_{1}\left(n B_{2}-B_{1}\right)\right]-J_{2}\left[A_{1}\left(n B_{2}-B_{1}\right)\right]\right\} \\
& \quad=2\left(n B_{2}-B_{1}\right) J_{1}\left[A_{1}\left(n B_{2}-B_{1}\right)\right] .
\end{aligned}
$$

FEL radiation is highly relevant to the longitudinal distribution of the electron beam, especially the beam current and the bunching parameter. After the DS2, attosecond scale microstructures come out over one seed wavelength [30] in the electron beam. This kind of electron beam will generate coherence APTs at the very beginning of the radiator. However, the ultrashort radiation structure will be quickly smeared out by the slippage effect in the long radiator when the period number is larger than the harmonic number. It is found that the radiation trains can be maintained and amplified by taking advantage of the mode-locked technique [8,21], as shown in Fig. 2. A series of spatiotemporal shifts are introduced between the radiation and the copropagating electron bunch by delaying the electron bunch using magnetic chicanes inserted between undulator modules. These small chicanes also act as dispersion sections, but the strengths of the chicanes $\left(R_{56} \sim 10^{-6} \mathrm{~m}\right)$ are much smaller than the strengths of the DSs $\left(R_{56} \sim 10^{-3} \mathrm{~m}\right)$. The total slippage per module is $\bar{s}=$ $\bar{l}+\bar{\delta}$, where $\bar{l}$ is the slippage due to the undulator and $\bar{\delta}$ is the slippage due to the chicane. In each module, the radiation wave front will propagate ahead and interact with the electrons within $\bar{l}$ in the undulator. After passing

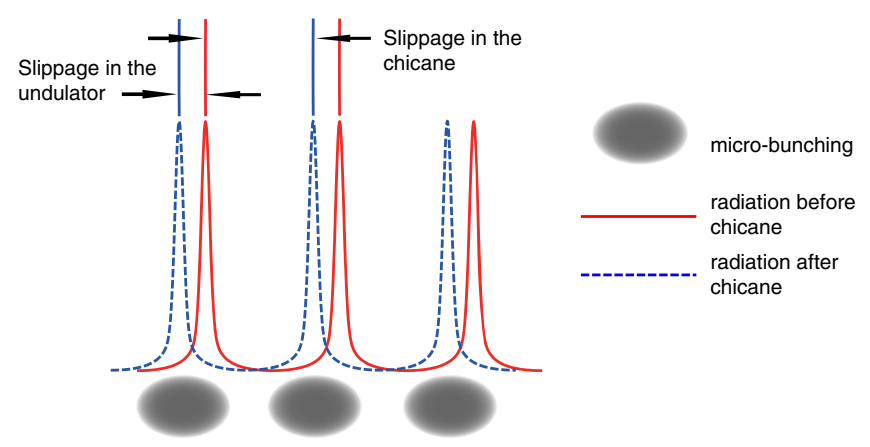

FIG. 2. Principle of the mode-locked amplification for a seeded FEL. through a small chicane, the radiation will be shifted ahead by $\bar{\delta}$ and interact with another part of the electron beam in the following undulator. When the total slippage length $\bar{s}$ is equal to the wavelength of the energy modulation envelope $\lambda_{s}$, the radiation would take over the next high current spike at the beginning of the next undulator section. The FEL interaction at different longitudinal positions along the beam will be periodically suppressed or enhanced, so the train of evenly spaced pulses will develop aligned with the regions of high beam current and large bunching factor.

For the $n$th harmonic radiation, the electrons in $[0,2 \pi]$ in the modulator are transformed to $[0,2 n \pi]$ in the radiator, and the bunching parameter corresponding to the radiation wavelength $\lambda_{r}=\lambda_{\text {seed } 1} / n$ in the radiator is no more uniform over the distance of one seed laser wavelength $\lambda_{\text {seed } 1}$. As the radiation only interacts with electrons within $\bar{l}$ in each module, the electrons over the distance $\lambda_{\text {seed } 1}$ should be uniformly divided into slices with length $\bar{l}$ and the electrons in each slice are independent with other slices. The local current $I_{n}$ and the local bunching factor $B_{n}$ at position $z$ along the electron beam should be defined as

$$
I_{n}(z)=\int_{z-\bar{l} / 2}^{z+\bar{l} / 2} N\left(z^{\prime}\right) d z^{\prime} / \bar{l},
$$

$$
B_{n}(z)=\int_{z-\bar{l} / 2}^{z+\bar{l} / 2} N\left(z^{\prime}\right) e^{-i n z^{\prime}} d z^{\prime} / \int_{z-\bar{l} / 2}^{z+\bar{l} / 2} N\left(z^{\prime}\right) d z^{\prime} .
$$

Generally, when the period number of the radiator is much larger than the harmonic number $n$, the local current and local bunching factor can be averaged over $\lambda_{\text {seed1 }}$. Then Eqs. (5) and (6) will reduce to the universal results reported in Ref. [12].

To illustrate the physics behind this triple-modulator scheme, the longitudinal phase space and corresponding local parameters distribution in one period of the energy modulation envelope $\lambda_{s}$ at the entrance of radiator are shown in Fig. 3. The frequency of seed 2 is chosen to be the same as seed $1, K_{1}=1$. The frequency of seed 3 is
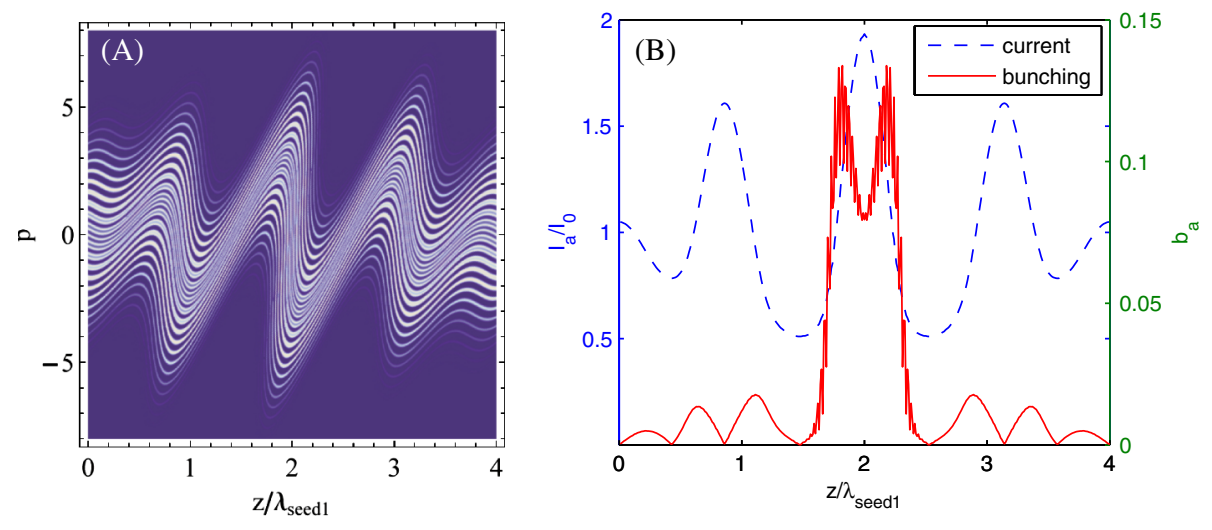

FIG. 3. Phase space (a) and corresponding local current factor and local bunching factor distributions (b) at the exit of DS2. 
different from seed1, $K_{2}=3 / 4$. For $n_{2}=n_{3}=20$, the optimized parameters that maximize the local bunching factor are $A_{1}=2, A_{2}=1.5, B_{1}=14.95, B_{2}=0.42$, and $\phi+\phi^{\prime}=\pi$. The harmonic number $n$ is 34 , which is a feasible value for the EEHG scheme.

As $K_{2}=3 / 4$, the phase space of the electron beam will be periodic with $\lambda_{s}=4 \lambda_{\text {seed } 1}$. The phase space in one period of energy modulation envelope is shown in Fig. 3(a), which is calculated by Eq. (2). The beam local current and local bunching factor distributions are calculated by Eqs. (5) and (6), and illustrated by Fig. 3(b). The local bunching factor in the central part of each period is around 0.12 ; while in other parts are around 0.01 . The peak value of the local current is nearly 2 times larger than the initial one.

\section{GENERATION OF INTENSE APTS}

To illustrate a possible application of the proposed scheme with realistic parameters, we take the nominal parameters of the upgraded Shanghai Soft X-ray FEL (SXFEL) project [29]. The main parameters of the upgraded SXFEL are presented in Table I. For comparison purposes, three cases are considered, including the conventional EEHG and triple-modulator scheme proposed here with seed 3 off and on. We assume that the wavelengths of seed 1 and seed 2 are $266 \mathrm{~nm}$ in these three cases, and the wavelength of seed 3 is chosen to be $355 \mathrm{~nm}$ for the third case. To minimize the transverse modulation variations, the rms transverse sizes of the laser beams are all set to be $1000 \mu \mathrm{m}$, which is nearly 10 times larger than that of the electron beam in the modulators. To maximize the bunching factor at the 34th harmonic of seed1, the optimized parameters are $A_{1}=2, A_{2}=3, B_{1}=13.2, B_{2}=$ 0.37 for the first two cases [14], and chosen to be the same as that used in Fig. 3 for the third case. The corresponding peak powers of the seed lasers used in the simulations are $P_{1}=15 \mathrm{MW}, P_{2}=32 \mathrm{MW}$ for the first two cases and $P_{1}=15 \mathrm{MW}, P_{2}=8 \mathrm{MW}, P_{3}=5 \mathrm{MW}$ for the third
TABLE I. Simulation parameters.

\begin{tabular}{lrcr}
\hline \hline Electron beam & & & \\
\hline Beam energy [GeV] & & 1.3 & \\
Slice energy spread [keV] & & 65 & \\
Peak current [A] & & 1500 & \\
Slice emittance [mm mrad] & & $\sim 0.5$ & \\
Pulse length (FWHM) [ps] & 1 & 2 & 355 \\
\hline Seed laser & 266 & 266 & 8 \\
\hline Wavelength [nm] & 8 & 8 & 3 \\
Pulse length (FWHM) [ps] & 1 & 2 & 8 \\
\hline Modulator & 8 & 8 & 10 \\
\hline Period length [cm] & 10 & 10 & \\
Period number & & & \\
Resonant wavelength [nm] & 266 & 266 & 355 \\
\hline Radiator & & 3 & \\
\hline Period length [cm] & & 7.8 & \\
Resonant wavelength [nm] & & 1.535 & \\
Undulator parameter $K$ & &
\end{tabular}

case. The resonant wavelengths of radiators are $\lambda_{r}=$ $7.8 \mathrm{~nm}$. For the second case, undulator periods in each module are chosen to be $N_{u}=8$ so that $\bar{l}=8 \lambda_{r}$, and the relative electron beam over radiation slippage induced by the dispersive effect of each chicane placed between the undulator modules is set to be $\bar{\delta}=26 \lambda_{r}$, with total slippage due to undulator and delay section $\bar{s}=\lambda_{\text {seed2 }}$. When seed 3 is used to generate the energy modulation envelope, the chicane delay is changed to $\bar{\delta}=128 \lambda_{r}$ which makes $\bar{s}=4 \lambda_{\text {seed2 }}$. The length of each chicane is $0.5 \mathrm{~m}$ with adjustable dispersion strength from 0 to $18 \mu \mathrm{m}$. External quads are inserted between undulator modules for every $1.8 \mathrm{~m}$. The average beta functions of the electron beam in the radiator are $\bar{\beta}_{x}=24.4 \mathrm{~m}$ and $\bar{\beta}_{y}=8.6 \mathrm{~m}$, respectively.
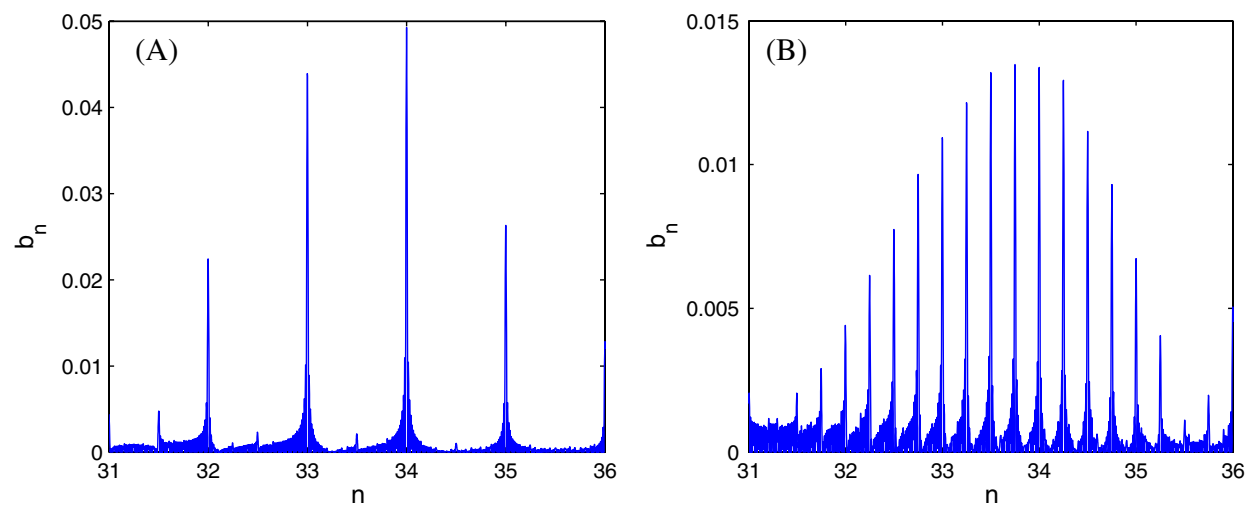

FIG. 4. Average bunching factor as a function of the harmonic number at the entrance of the radiator: (a) conventional EEHG and triple-modulator scheme with seed3 off; (b) triple-modulator scheme with seed3 on. 


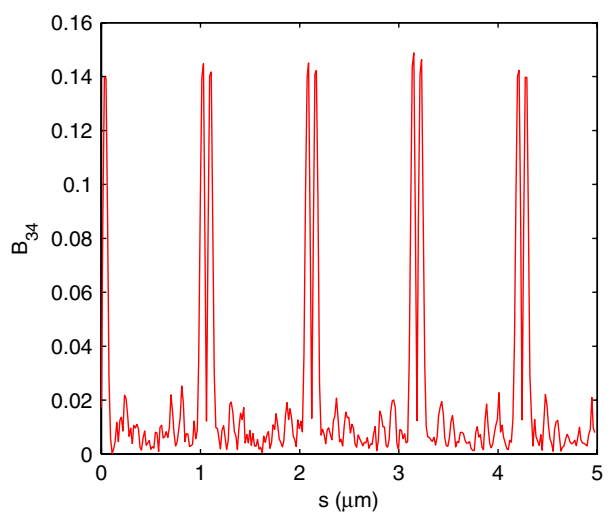

FIG. 5. Local bunching factor distribution along the electron beam at the entrance of the radiator for the third case.
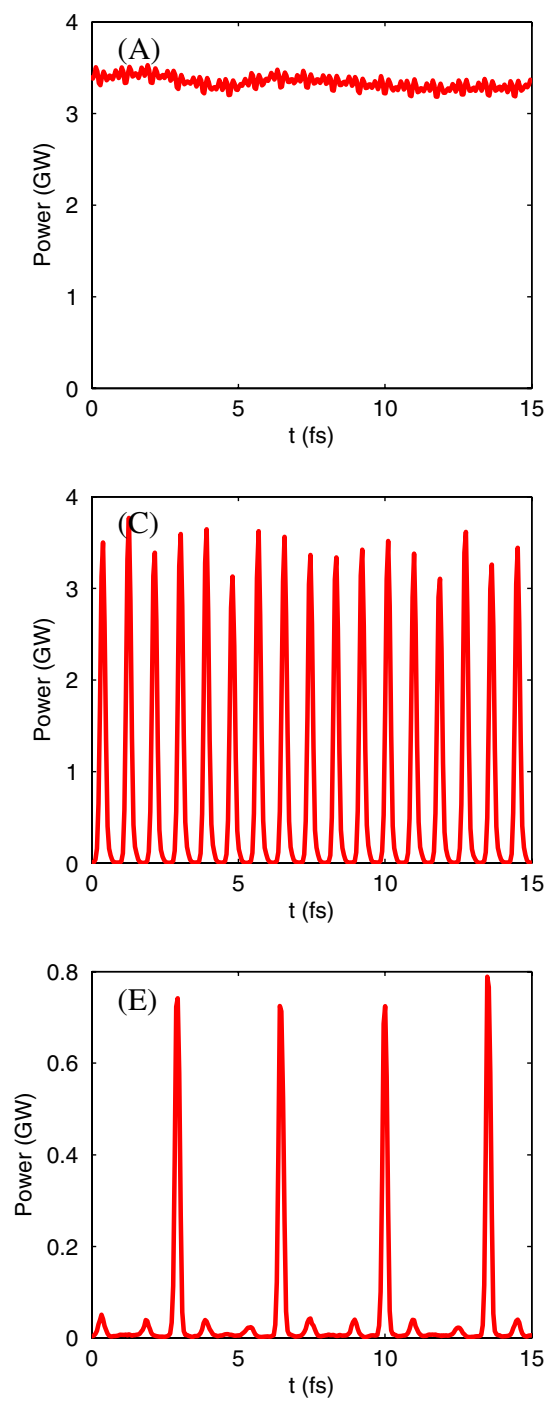

The simulation is carried out by the three-dimensional FEL code GENESIS [31], with a self-consistent method [32]. The local current distribution over the distance of one seed wavelength is also considered. After passing through DS2, the electron beam will be bunched at various harmonics of seed 2 as Fig. 4 shows, which indicates bunching at different modes. The bunching spectrums for the first two cases are the same with the maximal value of about 0.05 at the 34th harmonic [Fig. 4(a)]. The bunching spectrum of the third case contains more harmonics but a lower maximal value of about 0.015 [Fig. 4(b)]. By properly setting the parameters of the mode-locked radiator, some of these harmonics (modes) can be phase locked and amplified to saturation. The local bunching factor distribution calculated by GENESIS for the third case is shown in Fig. 5. One
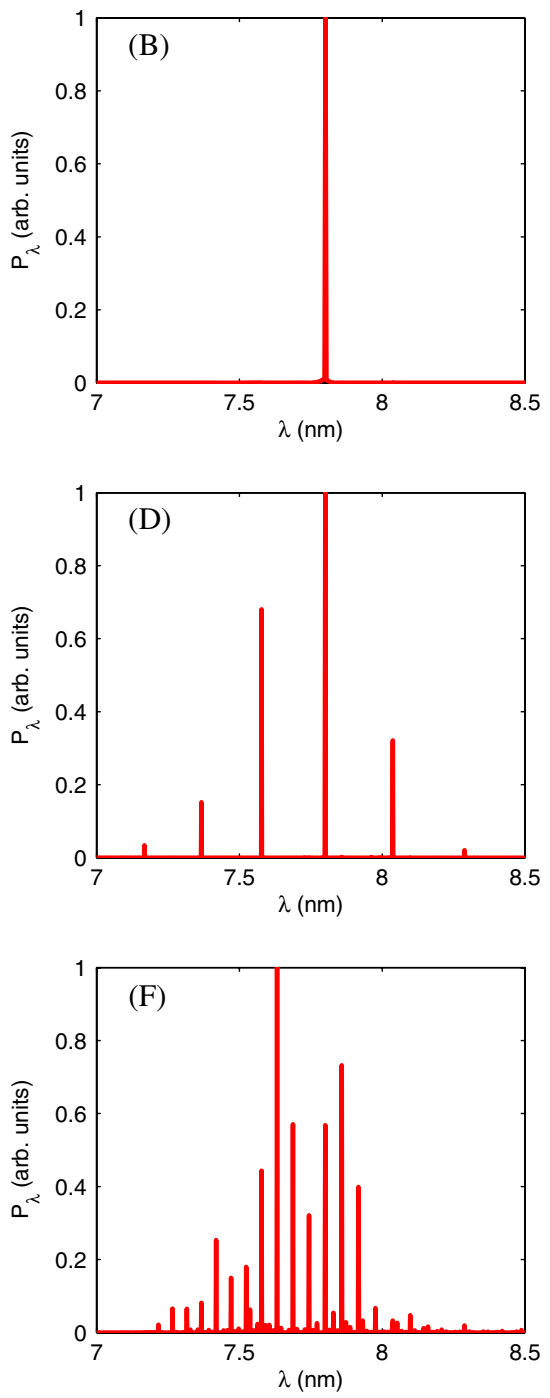

FIG. 6. Radiation pulses close to saturation and the corresponding radiation spectrums for three cases. (a) radiation pulse of conventional EEHG, (b) spectrum of conventional EEHG, (c) radiation pulse of triple-modulator scheme with seed3 off, (d) spectrum of triple-modulator scheme with seed3 off, (e) radiation pulse of triple-modulator scheme with seed 3 on, and (f) spectrum of triplemodulator scheme with seed3 on. 
can find that the maximal local bunching factor is nearly 1 order of magnitude larger than the average one, which is also much larger than the average bunching factor of the conventional EEHG.

The output radiation pulses and corresponding spectrums close to saturation for these three cases are shown in Fig. 6. For the conventional EEHG case, as shown in Fig. 6(a), the radiation power is constant along the chosen part of the electron beam with a spectrum centered at the resonant wavelength of Fourier transform-limited bandwidth [Fig. 6(b)], results typical of the seed FEL regime. In Fig. 6(c), mode locking occurs in the radiator, and stable separated pulse trains with width of about 200 as evenly spaced by about 800 as is generated. The spectrum [Fig. 6(d)] has sideband modes with separation of about $0.22 \mathrm{~nm}$. For the third case, when the third modulator is used to generate the energy modulation envelope, the separation between the radiation pulses will become 4 times larger than the second case as Fig. 6(e) shows. The 200 as radiation pulses are separated by about $3.3 \mathrm{fs}$, which can be tuned even larger by changing the wavelength of seed 3 and the strengths of the chicanes in the radiator. The spectrum has more sideband modes with separation of about $0.055 \mathrm{~nm}$ [Fig. 6(f)].

It has been mentioned in Ref. [8] that the delay chicanes will also introduce longitudinal energy dispersion and therefore enhance the FEL gain process. For the seeded FEL case studied here, a greater peak current will make the FEL gain length even shorter. Figure 7 shows the radiation peak powers as a function of the undulator length for these three cases. In order to illustrate the difference in FEL gain length, radiation power variations in the chicanes are not included in these curves. It is calculated from these curves that the gain

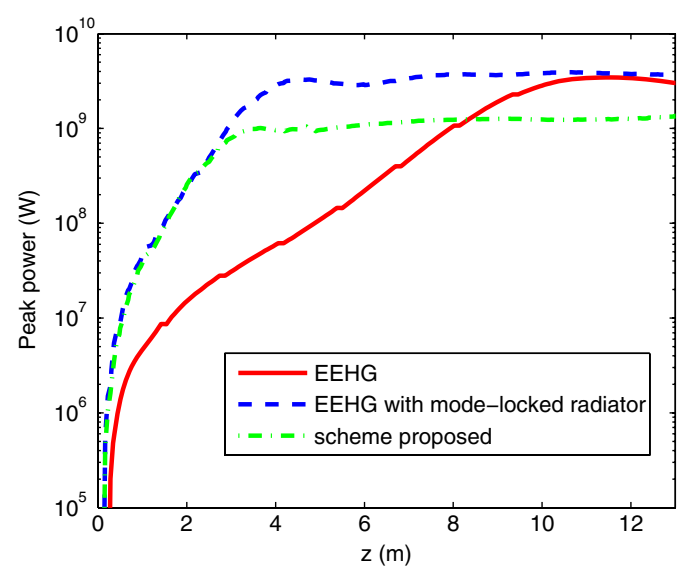

FIG. 7. Radiation peak power as a function of undulator length (radiation power variations in the chicanes of each module is not drawn) for the three cases: EEHG without a mode-locked radiator (red solid curve), triple-modulator scheme with seed3 off (blue dashed curve), and triple-modulator scheme with seed3 on (green dot-dashed curve). length for the conventional EEHG is $1.36 \mathrm{~m}$, which is reduced to $0.62 \mathrm{~m}$ when using the mode-locked radiators. The dispersion induced by the small chicanes will also accelerate the FEL saturation process and result in a shorter saturation length but a lower saturation power. It is found in Fig. 4 that the FEL reaches saturation after 15 modules with the saturation peak power of about $3.5 \mathrm{GW}$ for the second case. By increasing the strengths of the chicanes for the third case, an even shorter saturation length of about $3 \mathrm{~m}$ of undulator (11 modules) is expected, with the saturation peak power of about $0.8 \mathrm{GW}$. It should be pointed out that the mode-locked radiator will produce less average output power than the conventional radiator. It can be calculated from Figs. 6(a), 6(c), and 6(e) that the average saturation power is about 6 times smaller than the conventional radiator for the second case, and about 100 times smaller than the conventional radiator for the third case. So this kind of mode-locking undulator beam line should only be adopted when users are more interested in the APTs than the average output power.

\section{SOME PRACTICAL ISSUES THAT MAY AFFECT THE RADIATION}

As mentioned above, the spacing $\bar{s}$ between the APTs is determined by the period of energy modulation envelope $\lambda_{s}$ and the corresponding strength of the chicane in each module. The $\lambda_{s}$ can be tuned to be much longer than the radiation wavelength when the wavelengths of the seed lasers are very close to each other. However, the strength of the chicane $R_{56(c)}$ must be constrained to a small value to meet the requirement for the coherent bunching of the electrons in the radiator. The change in path length induced by the chicane should be smaller than a quarter of the radiation wavelength: $\Delta \delta=R_{56(c)} A \sigma_{E} / E<\lambda_{r} / 4$, where $A$ is the total dimensionless amplitudes of energy modulation at the entrance of the radiator. The maximal delay time is inversely proportional to the total energy spread of the electron beam. For the cases studied in Sec. III, the $R_{56(c)}$ should be smaller than $7.8 \times 10^{-6} \mathrm{~m}$, and thus the spacing between the attosecond pulses should be smaller than $13 \mathrm{fs}$.

There are a number of chicanes in the mode-locked radiator. As the strengths of these chicanes are relativity small (for the third case in Sec. III, the total $R_{56}$ induced by the chicanes in the radiator is only about $9.8 \times 10^{-7} \mathrm{~m}$, which is much smaller than the strength of DS2 $3.12 \times$ $10^{-4} \mathrm{~m}$ ), the coherent synchrotron radiation and incoherent synchrotron radiation effects can be neglected. However, the effects of field stability of these chicanes and energy jitter of the electron beam on the $\Delta z$ are not negligible. The electron beam delay can be written as $\delta=$ $e^{2} B^{2} L^{3} / \gamma_{0}^{2}$. The tolerances on the magnetic field and beam central energy stability should be limited to $\Delta B / B<\lambda_{r} / 2 \delta \sqrt{N_{c}}$ and $\Delta \gamma_{0} / \gamma_{0}<\lambda_{r} / 2 \delta \sqrt{N_{c}}$ to satisfy the radiation wavelength phase matching condition 
$\Delta z<\lambda_{r}$, where $N_{c}$ is the number of chicanes in the radiator. For the second and third cases studied in Sec. III, the criteria for the chicane stability are $\Delta B / B<$ $6.1 \times 10^{-3}, \Delta \gamma_{0} / \gamma_{0}<6.1 \times 10^{-3}$ and $\Delta B / B<7.8 \times$ $10^{-4}, \Delta \gamma_{0} / \gamma_{0}<7.8 \times 10^{-4}$, respectively, which are feasible with state-of-the-art accelerator technology.

The intensity jitters and phase errors of the seed lasers will also affect the property of the radiation pulses. The jitters of the laser powers will decrease the bunching factor and thus degrade the FEL performance. This effect has been considered by Eq. (6) for the third case in Sec. III. It is found from calculation that the maximal local bunching factor will drop from 0.14 to 0.12 when the shot-to-shot power fluctuations of the three seed lasers are $\pm 5 \%$, and the bunching factor will drop to about 0.03 when the power fluctuations are $\pm 10 \%$. So it is necessary to control the power fluctuations within $\pm 5 \%$ to maintain the bunching factor. The phase variation in the initial seed laser will be amplified by a factor of $n$ for the $n$th harmonic and will have a strong impact on the temporal properties of the output radiation $[33,34]$. The temporal properties of the radiation pulse can be quantified by the quality factor $M_{t}^{2}$, which is defined as the ratio between the time-bandwidth product for real and Fourier transform-limited pulse. Generally, $M_{t}^{2}=\sqrt{1+n_{t}^{2} \alpha^{2}}$, where $\alpha$ is the frequency chirp in the initial seed laser and $n_{t}$ is the total harmonic number. For the cases studied in Sec. III, all the seed lasers are generated from the $800 \mathrm{~nm}$ Ti:sapphire laser, so the total harmonic number $n_{t}$ is 102 . To make the bandwidth of the output radiation smaller than 10 times of the Fourier transform limited, the frequency chirp $\alpha$ must be controlled to be smaller than 0.1, which means that the relative discrepancy of the time-bandwidth product of the $800 \mathrm{~nm}$ laser pulse from the ideal transform-limited performance should be no larger than $1 \%$.

\section{CONCLUSIONS}

In summary, a novel scheme for generation of attosecond pulse trains is proposed and studied analytically and numerically. Unlike a SASE amplifier operating in the short-pulse mode, the output radiation of a mode-locked seeded FEL will be much more stable in both its timing and power with state-of-the-art accelerator technologies. Simulation results show that trains of 200 as x-ray pulses with GW level peak power can be generated directly from UV seed lasers using the proposed scheme. The spacing between the output short pulses can be tuned to be much longer than the seed laser wavelengths. Some practical limiting factors that might affect the performance of the proposed scheme are also taken into account, and it is shown that these effects can be controlled within an acceptable level with the present technologies. This type of mode-locked seeded FEL is potentially useful where stable APTs are desired. It may open a new regime of ultrafast sciences, such as real time observation and control of atomic-scale electron dynamics. Further investigations on its potential applications will be performed in the future.

\section{ACKNOWLEDGMENTS}

The authors would like to thank L. Shen, H. X. Deng, T. Zhang, Dong Wang, and Dao Xiang (SLAC) for helpful discussions and comments. This work is supported by Major State Basic Research Development Program of China (973 Program) (Grant No. 2011CB808300) and National Natural Science Foundation of China (Grant No. 10935011).

[1] E. L. Saldin, E. A. Schneidmiller, and M. V. Yurkov, Opt. Commun. 212, 377 (2002).

[2] E. L. Saldin, E. A. Schneidmiller, and M. V. Yurkov, Opt. Commun. 239, 161 (2004).

[3] A. A. Zholents and G. Penn, Phys. Rev. ST Accel. Beams 8, 050704 (2005).

[4] E. L. Saldin, E. A. Schneidmiller, and M. V. Yurkov, Phys. Rev. ST Accel. Beams 9, 050702 (2006).

[5] A. A. Zholents and M. S. Zolotorev, New J. Phys. 10, 025005 (2008).

[6] P. Emma, K. Bane, M. Cornacchia, Z. Huang, H. Schlarb, G. Stupakov, and D. Walz, Phys. Rev. Lett. 92, 074801 (2004).

[7] S. Reiche, P. Musumeci, C. Pellegrini, and J. B. Rosenzweig, Nucl. Instrum. Methods Phys. Res., Sect. A 593, 45 (2008).

[8] N.R. Thompson and B.W. J. McNeil, Phys. Rev. Lett. 100, 203901 (2008).

[9] A. A. Zholents and W. M. Fawley, Phys. Rev. Lett. 92, 224801 (2004).

[10] D. Xiang, Z. Huang, and G. Stupakov, Phys. Rev. ST Accel. Beams 12, 060701 (2009).

[11] H.X. Deng and Z.M. Dai, Chinese Phys. C 34, 1140 (2010).

[12] L. H. Yu, Phys. Rev. A 44, 5178 (1991).

[13] G. Stupakov, Phys. Rev. Lett. 102, 074801 (2009).

[14] D. Xiang and G. Stupakov, Phys. Rev. ST Accel. Beams 12, 030702 (2009).

[15] P. Johnsson et al., Phys. Rev. Lett. 95, 013001 (2005).

[16] T. Remetter et al., Nature Phys. 2, 323 (2006).

[17] J. Mauritsson, P. Johnsson, E. Mansten, M. Swoboda, T. Ruchon, A. L'Huillier, and K. J. Schafer, Phys. Rev. Lett. 100, 073003 (2008).

[18] K. P. Singh et al., Phys. Rev. Lett. 104, 023001 (2010).

[19] M. Swoboda, T. Fordell, K. Klünder, J. M. Dahlström, M. Miranda, C. Buth, K. J. Schafer, J. Mauritsson, A. L'Huillier, and M. Gisselbrecht, Phys. Rev. Lett. 104, 103003 (2010).

[20] K. Klünder et al., Phys. Rev. Lett. 106, 143002 (2011).

[21] E. Kur, D. J. Dunning, B. W. J. McNeil, J. Wurtele, and A. A. Zholents, New J. Phys. 13, 063012 (2011).

[22] D. J. Dunning, B. W. J. McNeil, N.R. Thompson, and P. H. Williams, Phys. Plasmas 18, 073104 (2011).

[23] D. Xiang et al., Phys. Rev. Lett. 105, 114801 (2010). 
[24] D. Xiang et al., Phys. Rev. Lett. 108, 024802 (2012).

[25] Z. T. Zhao, in Proceedings of FEL11, Shanghai, China (2011), WEOBI2 (unpublished).

[26] Z. T. Zhao et al., Nature Photon. 6, 360 (2012).

[27] K. E. Hacker, S. Khan, G. Angelova Hamberg, V. G. Ziemann, A. Azima, P. Salén, P. van der Meulen, and H. Schlarb, in Proceedings of FEL11, Shanghai, China (EPSAG, China, 2011), TUPB10.

[28] G. Penn and M. Reinsch, J. Mod. Opt. 58, 1404 (2011).

[29] Z.T. Zhao et al., in Proceedings of IPAC2011, San Sebastián, Spain (EPS-AG, Spain, 2011), THPC053.
[30] M.S. Sears, E. Colby, R. Ischebeck, C. McGuinness, J. Nelson, R. Noble, R. H. Siemann, J. Spencer, and D. Walz, Phys. Rev. ST Accel. Beams 11, 061301 (2008).

[31] S. Reiche, Nucl. Instrum. Methods Phys. Res., Sect. A 429, 243 (1999).

[32] J. Yan, M. Zhang, and H.X. Deng, Nucl. Instrum. Methods Phys. Res., Sect. A 615, 249 (2010).

[33] G. Geloni, V. Kocharyan, and E. Saldin, arXiv: $1111.1615 \mathrm{v} 1$.

[34] D. Ratner, A. Fry, G. Stupakov, and W. White, Phys. Rev. ST Accel. Beams 15, 030702 (2012). 